\title{
O ENVELHECIMENTO E O IDOSO NO ENSINO DE GRADUAÇÃO EM ENFERMAGEM NO BRASIL: DO PANORAMA ATUAL À UMA PROPOSTA DE CONTEÚdO PROGRAMÁTICO
}

\author{
THE AGING AND THE ELDERLY IN THE NURSING \\ UNDERGRADUATE IN BRAZIL: \\ FROM THE CURRENT PANORAMA TO A PROPOSAL CONTENT OF PROGRAM
}

\author{
Maria José Delboux Diogo* \\ Yeda Aparecida de Oliveira Duarte**
}

DIOGO, M.J.D., DUARTE, Y.A.O. O envelhecimento e o idoso de graduação em enfermagem no Brasil: do panorama atual à uma proposta de conteúdo programático. Rev. Esc. Enf. USP, v. 33, n. 4, p. 370-6, dez. 1999.

\section{DTS}

Foram verificados 77 cursos de graduação em enfermagem existentes no Pats quanto ao ensino de temas relacionados à atenção ao idoso, sua carga horária e tempo de inserção curricular. As autoras sugerem alguns temas considerados básicos para a formação de enfermeiros que venham a atuar com idosos nos diferentes contextos de atenção à saúde.

UNITERMOS: Enfermagem geriátrica. Ensino. Educação em Enfermagem.

\section{T.T.}

The teaching nursing undergraduate was verified in 77 courses in Brazil about elderly's attention, hourly load and time of insert of this content in the curriculum. The authors suggest fundamental themes to the nurses' formation to act with elderly in the different contexts in their health's attention.

UNITERMS: Geriatric Nursing. Teaching. Education, nusing.

\section{INTRODUÇÃO}

Pesquisas desenvolvidas nos últimos anos vem demonstrando que a população mundial está envelhecendo e que este parece ser um fenômeno irreversível, ou seja, o número de pessoas idosas vem se tornando cada vez mais representativo do ponto de vista numérico quando são analisadas as populações totais ${ }^{(13,16)}$.

A Organização Mundial de Saúde estabelece que um país pode ser considerado estruturalmente envelhecido quando sua taxa de gerontes ultrapassa $7 \%$ da população total. Em 1980, o Brasil possuía uma cifra de 6,3\% de idosos em sua população geral. Estima-se que no ano 2000 este total ultrapasse os $8 \%$ chegando a $13,8 \%$ em $2025^{(14,20,22,25,26,27)}$.

Estas constatações são acompanhadas de importantes preocupações pois sabe-se que apesar do envelhecimento ser um processo natural, ele não ocorre de forma homogênea a todos os indivíduos e assim a longevidade faz-se acompanhar por demandas específicas que necessitam ser reconhecidas, avaliadas e atendidas por profissionais devidamente capacitados. Dentre estes, sem dúvida a enfermagem ocupa local de destaque ${ }^{(5,26)}$. A forma como tais questões vem sendo trabalhadas na formação dos futuros profissionais desta área motivou o desenvolvimento deste estudo.

\section{Aspectos Demográficos e Epidemiológicos do} Envelhecimento Populacional

O envelhecimento populacional, embora seja um fenômeno universal, no Brasil apresenta características peculiares devido à velocidade em que se dá esta transição

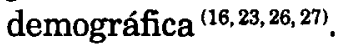

\footnotetext{
- Enfermeira. Professor Assistente Doutor do Departamento de Enfermagem da Faculdade de Ciências Médicas da UNICAMP. E-mail: adiogo@theway.com.br

- Enfermeira. Professor Assistente da Escola de Enfermagem da USP. E-mail: yeda@uol.com.br
} 
Nos últimos cem anos o número de idosos vem aumentando em todo o mundo devido a três fatores principais:

- redução da mortalidade geral e em especial a infantil

diminuição das taxas de fecundidade

aumento das taxas de sobrevida ou expectativa de vida

No Brasil, este último aspecto merece ser destacado. A expectativa de vida dos brasileiros duplicou ao longo deste século passando de 33,7 anos em 1900 para 63,5 anos em 1980 com projeções de 75,3 anos em $2025^{(27)}$.

Esta transição demográfica vem sendo acompanhada pela transição epidemiológica ou seja, onde antes as principais causas de morte da população estavam relacionadas às doenças infecciosas e parasitárias atualmente observa-se a presença cada vez mais significativa de doenças crônico-degenerativas e outros agravos gerados pelo próprio homem (homicídios, acidentes, etc) ${ }^{(10,17,18,25)}$.

Ainda nesta perspectiva verifica-se que o Brasil que ocupava em 1950 o $16^{\circ}$ lugar na escala mundial em número de idosos ou seja, 2,1 milhões de pessoas com mais de sessenta anos, passará em 2025 para $6^{\circ}$ lugar com a espantosa perspectiva de 32 milhões de gerontes no total de sua população. Isto representa o maior aumento proporcional de idosos entre os países mais populosos do mundo no mesmo período; assim o número de gerontes aumentará quinze vezes enquanto a população como um todo aumentará somente $\operatorname{cinco}^{(2,26,27)}$.

Assim sendo, estudos gerontológicos que busquem estratégias para 0 atendimento às demandas específicas emanadas por esta faixa etária da população bem como o desenvolvimento de intervenções adequadas devem ser estimulados pois o idoso não pode ser considerado um "velho adulto"; ao contrário, são indivíduos que requerem abordagens específicas advindas de conhecimentos profundos dos efeitos bio-psico-socio-culturais do envelhecimento sobre os seres humanos ${ }^{(5,6)}$.

Muitos avanços, no entanto, foram alcançados no sentido de proporcionar melhorias na qualidade assistencial dos idosos. $\mathrm{Na} 8^{a}$. Conferência Nacional de Saúde, em 1988, foi apresentado o Programa sobre Política de Envelhecimento ${ }^{(4)}$. Este marco inicial culminou com a elaboração da Lei n. 8.842 de 4 de janeiro de 1994 que dispõe sobre a Política Nacional do Idoso e cria o Conselho Nacional do Idoso ${ }^{(3)}$. Esta Lei foi regulamentada em $1^{\circ}$ de julho de 1996 e mais recentemente gerou o Plano de Ação Governamental integrado para o desenvolvimento da Política Nacional do Idoso concretizando assim, ao menos legislativamente, as intenções dos estudiosos deste campo. Neste último documento bem como na referida lei, nas áreas relativas à educação e saúde são sugeridas recomendações no sentido de habilitar os profissionais voltados ao atendimento desta população através de programas educativos de graduação e pós graduação além de treinamentos específicos para a atualização e capacitação continuada das equipes profissionais de saúde envolvidas na gerência, planejamento, pesquisa e assistência aos idosos.

A enfermagem, enquanto elemento integrante da equipe de saúde, deve estar devidamente habilitada para poder atuar mais adequadamente junto a esta população através da inserção de conteúdos gerontogeriátricos nos currículos de graduação, pós graduação, especialização e também nos programas de educação continuada dos serviços de enfermagem já implantados.

\section{O Ensino de Enfermagem Gerontogeriátrica nos Currículos de Graduação}

Conteúdos relativos ao ensino de enfermagem geronto-geriátrica foram inseridos nos cursos de graduação em enfermagem ao longo do tempo e a partir da iniciativa de algumas escolas, independentemente da legislação vigente.

Em estudo realizado por ANGERAMI et $\mathrm{al}^{(1)}$, sobre a nomenclatura das matérias e disciplinas constantes no currículo mínimo de graduação segundo a Resolução CFE / n. 4/72 $2^{(4)}$ em comparação com a utilizada pelas Escolas de Enfermagem da região Sudeste do Brasil, observa-se a ausência de referências quanto ao ensino relacionado ao envelhecimento e ao idoso especificamente, embora os autores tenham mencionado que os "programas especiais incluem Saúde da Mulher, Trabalhador, Adulto e outros".

A enfermagem gerontológica é uma área praticamente nova no ensino de graduação ${ }^{(11)}$ embora desde 1966 tenha sido declarada uma especialidade pela American Nurses Association (ANA). A Divisão de Enfermagem Gerontológica desta Associação descreveu, em 1981, um programa para o ensino de graduação que previa conhecimentos básicos e o desenvolvimento de habilidades em enfermagem (incluindo enfermagem ao idoso) necessários para preparar um enfermeiro generalista para a prática em enfermagem gerontológica ${ }^{(28)}$.

A ORGANIZAÇÃO PANAMERICANA DE SAÚDE ${ }^{(23)}$ ao descrever as recomendações para o ensino de enfermagem geronto-geriátrica enfatiza que não se deve oferecer conteúdos parciais integrados a outras disciplinas para não correr o risco de dilui-los ou reduzilos ao longo da disciplina. Recomenda que os conteúdos sobre o cuidado ao idoso sadio precedam os referentes ao cuidado destinado ao idoso enfermo ou institucionalizado. Desse modo é permitido ao estudante a oportunidade de rever possíveis atitudes negativas sobre a velhice, assim como visualizar esta etapa da vida como um período de desenvolvimento e bem-estar apesar de poderem estar presentes limitações inerentes à idade Esta preocupação é pertinente pois 
os jovens podem trazer consigo conceitos pré-estabelecidos que na maioria das vezes se alteram após o contato com conteúdos teóricos e práticos relacionados ao idoso.

DeLORA; MOSES ${ }^{(7)}$, em estudo realizado com 201 estudantes de enfermagem sobre suas preferências quanto às especialidades relacionadas à atuação profissional, verificaram que somente $7(3,4 \%)$ gostariam muito de trabalhar em geriatria; $30(14.7 \%)$ gostariam; $53(26,0 \%)$ tinham sentimentos neutros; $52(25,5 \%)$ não gostariam; $52(25,5 \%)$ não gostariam muito; $6(2,9 \%)$ não sabiam e $4(2,0 \%)$ não responderam. Do total, 137 estudantes $(69,2 \%)$ responderam ainda que tinham interesse mínimo pelos pacientes com 60 anos ou mais. Ainda neste estudo, os autores investigaram os adjetivos, dentro de uma lista de 17, que melhor descreviam os sentimentos dos estudantes para com as diferentes especialidades da enfermagem e verificaram que 114 $(29,8 \%)$ respostas relacionavam-se a sentimentos de depressão, $60(15,7 \%)$ para lentidão, $47(12.3 \%)$ para fraqueza, entre outros, demonstrando a presença de estereótipos na imagem dos idosos elaborada pelos estudantes. Concluem os autores que uma das alternativas para alterar este quadro é a inserção no currículo de conteúdos relativos a esta temática.

A importância da inclusão de temas relacionados à Assistência de Enfermagem Gerontológica e Geriátrica na formação do enfermeiro foi verificada por DUARTE ${ }^{(8)}$ junto a enfermeiros que trabalham em hospitais, os quais referiram em sua maioria $(95 \%)$ ser necessária essa inclusão para a melhoria da assistência de enfermagem.

Frente ao exposto este estudo foi desenvolvido com a finalidade de oferecer subsídios para discussões e o desenvolvimento de outras pesquisas sobre a formação do enfermeiro em nível de graduação no que se refere a área de gerontologia.

\section{OBJETIVO}

Verificar o panorama do ensino de enfermagem geronto-geriátrica nos cursos de graduação em enfermagem no Brasil.

\section{MATERIAL E MÉTODO}

Para a realização deste estudo descritivo foram enviados questionários, em outubro de 1995 com o prazo de devolução estipulado em dezembro do referido ano, para todas as instituições de ensino do Brasil que continham curso de graduação em enfermagem, segundo o cadastro da Escola de Enfermagem da USP de setembro de 1995. Do total de 99 formulários enviados obteve-se 71 respostas $(71,72 \%)$.
O instrumento utilizado foi composto por questões abertas e fechadas, relacionadas ao ensino de enfermagem geronto-geriátrica quanto a:

- forma e ano de inserção desta temática no currículo; - carga horária específica e

- conteúdos ou temas abordados.

\section{RESULTADOS E DISCUSSÃo}

Nos 71 questionários devolvidos observou-se que apenas $8(11,26 \%)$ instituições referiram não abordar conteúdos relativos ao tema especificado.

No que se refere a inclusão de conteúdos curriculares sobre a atenção ao idoso, das 63 escolas que responderam afirmativamente, somente 14 ofereciam este conteúdo em disciplina específica, ao passo que 52 referiram inseri-1o em aulas de outras disciplinas; 9 em cursos extra-curriculares e 11 sob diferentes abordagens. Cabe ressaltar que das 63 instituições foram obtidas 86 respostas à questão, uma vez que esta abordagem poderia ocorrer nas diferentes formas apresentadas, como por exemplo uma mesma instituição oferecendo além de disciplina específica, aulas inseridas em outras disciplinas e cursos extracurriculares.

YURCHUCK; BROWER (28) descrevem um estudo realizado com 197 programas de graduação em enfermagem onde revelou-se um escasso preparo dos docentes no que se referia à atenção ao idoso. Acrescentam os autores que a falta de pessoas preparadas em enfermagem gerontológica foi mencionado como o maior obstáculo para a inserção de conteúdos sobre este tema seguido por falta de tempo, competição com outras especialidades e falta de interesse.

De fato, estes problemas também ocorrem em nossas escolas. Observa-se na maioria das vezes, iniciativas individuais de professores que, sensibilizados ou conscientes da problemática atual relacionada ao crescente número de idosos na população e as conseqüências sociais dessa explosão demográfica, acabem por inserir temas em sua área de conhecimento ou em disciplinas sob sua responsabilidade.

Outrossim as escolas de enfermagem do Brasil, ao que tudo indica, apresentam escasso número de professores adequadamente preparados, ou seja, envolvidos em programas de pós-graduação (mestrado e doutorado), desenvolvendo pesquisas ou estudando enfermagem gerontológica. A maioria dos professores dos cursos de graduação em enfermagem ensinam conteúdos sobre este tema por meio de programas estabelecidos através da sua experiência ou conhecimentos ou ainda por meio de auto-estudo e educação contínua. 
Johnson e Connelly apud YURCHUCK; BROWER ${ }^{(28)}$ descrevem alguns dos problemas relacionados ao preparo inadequado dos professores para o ensino de enfermagem geronto-geriátrica:

- o não reconhecimento da necessidade de conhecimentos e habilidades específicas para o cuidado de pacientes idosos por parte de professores de outras áreas de conhecimento ou especialização;

- a auto-aprendizagem ou o ensino com base em experiências pessoais que não asseguram o conhecimento adequado de gerontologia para o docente e muito menos o conhecimento a ser transferido ao aluno;

- a exposição de conceitos sobre promoção de saúde e prevenção de doenças em idosos com base somente em seus estudos individuais.

Em relação à carga horária destinada ao tema observou-se uma distribuição muito heterogênea entre as instituições variando de 2 a 180 horas com uma média de 34,27 horas e uma mediana de 20 horas. Ao considerar-se o limite de $\mathbf{1 5}$ horas para a obtenção de um crédito, e assim o mínimo necessário para compor uma disciplina, foi possível verificar que 40 instituições $(63,49 \%)$ poderiam oferecer disciplina específica sobre este assunto pois a carga horária referida comportaria tal divisão.

MENDES ${ }^{(21)}$, em sua pesquisa sobre o ensino de enfermagem geriátrica e gerontológica nos cursos de graduação realizado no final da década de 80 , verificou que dos 81 cursos estudados na ocasião, 9(11,11\%) desenvolviam disciplinas específicas relativas a esta temática sendo $4(4,93 \%$ ) optativas; 62 cursos $(76,54 \%)$ ofereciam tópicos inseridos em outras disciplinas regulares do tronco profissional curricular e 10(12,34\%) não haviam introduzido o assunto no ensino de graduação. As cargas horárias não foram claramente especificadas, porém não excediam 20 horas no semestre. A autora concluiu na ocasião, ser ainda muito pequena a contribuição do ensino de enfermagem para a formação de profissionais capacitados a assistir adequadamente a população idosa e apresentou uma proposta para o mesmo.

Quanto aos temas abordados no ensino sobre atenção ao idoso, obtivemos 294 respostas que foram categorizadas, por similaridade, em sete grandes grupos, a saber:

- Senescência: aspectos gerais e específicos $(N=90)$

- Enfermagem em geriatria e gerontologia $(\mathrm{N}=63)$

- Aspectos político-sociais, econômicos e culturais do envelhecimento $(\mathrm{N}=52)$

- Senilidade $(\mathrm{N}=37)$

- Atenção à saúde do idoso em diferentes contextos $(\mathrm{N}=29)$

- Aspectos demográficos e epidemiológicos $(\mathrm{N}=14)$

- O Enfoque multidisciplinar no ensino de graduação em enfermagem $(\mathrm{N}=9)$
Observa-se que houve predominância nos conteúdos relacionados à senescência ou seja, 90 respostas $(30,61 \%)$, enquadraram-se na categoria referente às alterações normais que ocorrem no processo de envelhecimento. Isto parece demonstrar que existe uma preocupação docente em descrever o envelhecimento como um processo natural do desenvolvimento humano e assim evitar dois tipos de erros muito freqüentes conforme citado por JACOB FILHO; SOUZA ${ }^{(15):}$ " todas as alterações encontradas em um idoso serem erroneamente atribuidas ao seu envelhecimento natural, o que impediria a deteç̧ão de processos patológicos passíveis de tratamento ou mesmo de cura" e "sinais e sintomas explicados pela senescência serem equivocadamente atribuídos a doenças, determinando a realização de exames $e$ tratamentos desnecessários. Em suma, o próprio envelhecimento sendo tratado como doença".

Observa-se que o segundo tema predominantemente mencionado, isto é, em 63 respostas $(21,43 \%)$, refere-se à enfermagem geronto-geriátrica o qual engloba a assistência de enfermagem em diferentes contextos (asilo, ambulatório, hospital e domicílio). Esses dados parecem demonstrar que os professores dos cursos de enfermagem estão preocupados com a formação dos enfermeiros para a assistência aos idosos em diferentes âmbitos, o institucional e o não institucional, representando uma tendência mundial. Esta preocupação vai ao encontro da proposta da Organização Mundial de Saúde que recomenda atividades educativas voltadas para as diferentes opções de serviços médicos e sociais de atenção ao idoso.

Em 52 respostas (17,69\%) foi possível perceber a preocupação em contextualizar o envelhecimento num panorama mais amplo do que o estritamente biológico discutindo abordagens político-sociais, econômicas e culturais relativas a esta faixa etária o que vem ao encontro de todos os discursos gerontológicos ${ }^{(11,12,20,21,23,24,28)}$.

A senilidade, segundo o número de respostas obtidas é um tema valorizado por 37 escolas de enfermagem (12,59\%). Embora a abordagem da maioria destas instituições se restrinja às doenças mais comuns nesta faixa etária, três delas responderam ainda abordarem conteúdos relacionados a hábitos prejudiciais à saúde que provocam dependência e a deficiência no idoso (física e mental).

Em relação aos tópicos mencionados, houve $29(9,86 \%)$ referências quanto ao ensino de conteúdos abordando a atenção ao idoso em diferentes contextos(educação em saúde, institucionalização, atenção domiciliária e outros). Este conjunto de respostas foi destacado da categoria "aspectos de enfermagem geronto-geriátrica" por não fazerem referência específica à assistência de enfermagem nestas situações. Assim, percebe-se que alguns dos grandes problemas relacionados ao idoso dentre eles, quedas, cuidadores e institucionalização também são 
abordados durante a formação do enfermeiro porém, não de forma expressiva, apesar de sua significativa importância nesta área.

Houve apenas 14 referências $(4,76 \%)$ sobre conteúdos relacionados aos aspectos demográficos e epidemiológicos, fato este que causa surpresa, uma vez que tais tópicos deveriam embasar todo planejamento assistencial a qualquer demanda populacional

Apenas 9 referências $(3,06 \%)$ citavam o enfoque multidisciplinar como conteúdo a ser abordado no ensino de graduação, o que causa certa preocupação pois a abordagem geronto-geriátrica eficiente é predominantemente realizada pela integração dos diferentes campos de conhecimentos das diversas áreas de atuação que objetivam a melhoria da qualidade de vida do idoso.

Outrossim cabe ressaltar que as categorias descriminadas não correspondem a temática abordada por todas as instituições pois as mesmas, em nenhum momento, apresentaram similaridade em seu eixo condutor o que gera a formação diferenciada de profissionais da mesma área de atuação num país já tão heterogêneo.

Ao compararmos estes resultados com os obtidos por MENDES ${ }^{(21)}$, não foram observados grandes avanços e até alguns retrocessos uma vez que muitos tópicos foram suprimidos o que demonstra um desencontro com as atuais demandas socio-epidemiológicas. Naquela ocasião a autora descreveu que dentre os principais temas abordados destacaram-se:

- Conceitos e fundamentos de Gerontologia e Geriatria em Enfermagem Geriátrica

- Processo de envelhecimento normal: alteraçóes biológicas nos órgãos

- Necessidades e problemas de saúde dos idosos - ênfase à cronicidade das doenças, incapacidades progressivas, intervenções cirúrgicas e terminalidade

- Uso de métodos para sistematizar a assistência de enfermagem, enfocando a terapêutica clínica, cirúrgica, condutas nutricionais e reabilitação.

Estudos similares, visto a tendência mundial do envelhecimento populacional, foram realizados em outros países. Os Estados Unidos(12, 19), por considerarem os enfermeiros como os responsáveis em prover a qualidade do cuidado à saúde dos idosos, reconhecem a necessidade de uma formação básica em enfermagem gerontológica para estes profissionais a fim de torná-los habilitados a atender adequadamente esta clientela. Referem ainda as dificuldades na inclusão de tais conteúdos pois, como citado por HOLTZEN et $\mathrm{al}^{(12)}$; gerontologia não é uma especialidade tradicional. Os profissionais de saúde incluindo os enfermeiros, geralmente não visualizam os idosos com necessidades diferentes dos demais adultos e, consequentemente, os estudantes não são estimulados a aplicarem conhecimentos ou conceitos específicos relacionados à gerontologia em sua dinâmica assistencial.
No Canadá, estudo realizado por EARTHY ${ }^{(9)}$ sobre o currículo gerontológico nos programas de graduação em enfermagem do país, aponta para a preocupação nacional com as rápidas mudanças demográficas, sociais e econômicas de sua sociedade o que tem favorecido modificações no planejamento global da assistência aos idosos. Os enfermeiros, continua a autora, são os primeiros profissionais de saúde a atuarem especificamente nesta demanda e assim sendo, salienta a necessidade de uma reestruturação em sua educação básica.

As respostas referentes à questão relativa ao ano de inserção dos conteúdos geronto-geriátricos nos currículos de graduação em enfermagem podem ser observadas no gráfico 1 :

\section{GRAFICO 1: ANO DE INSERCÃO DA TEMÁTICA RELATIVA A ATENĈ̃O AO IDOSO NOS CURRÍCULOS DAS INSTITUIÇÕES QUE AFIRMARAM ABORDAR ESTE TEMA, 1996.}

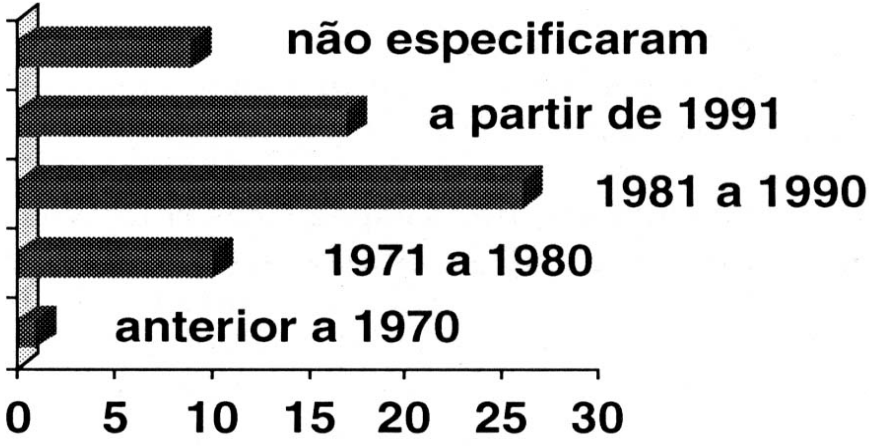

Conforme pode ser observado no Gráfico 1, a maioria das escolas inseriu em seu currículo conteúdos referentes ao envelhecimento e ao idoso na década de 80 , seguido da década de 90 , o que coincide com o período em que ocorreu um incremento nas discussões e estudos principalmente os demográficos e epidemiológicos referentes ao envelhecimento mundial e suas conseqüências para as diferentes sociedades.

Estes dados, quando comparados com os obtidos por MENDES ${ }^{(21)}$, parecem estar relacionados pois a autora verificou na ocasião de seu estudo (final da década de 80 ) que somente $10(12,34 \%$ ) dos 81 cursos estudados, não haviam inserido esta temática nos currículos de graduação, coincidindo portanto com o período de maior freqǚrncia das respostas das instituições. 


\section{CONCLUSÕES}

Conforme descrito anteriormente, o objetivo deste estudo era verificar a atual situação do ensino de graduação em enfermagem geronto-geriátrica no Brasil tendo o mesmo sido atingido. Foi possível constatar que das 71 instituições que colaboraram com o estudo, $63(87,73 \%)$ delas abordavam de alguma forma conteúdos relacionados a esta temática em seus currículos, porém de modo muito heterogêneo, não somente quanto a forma de inserção, mas também em relação a carga horária e aos temas abordados, o que sem dúvida gera a colocação no mercado de trabalho de profissionais com diferentes habilitações para assistir aos idosos.

É no entanto gratificante verificar que esta temática, de alguma forma já corresponde a uma preocupação da enfermagem.

\section{CONSIDERAÇÕES FINAIS}

As autoras deste estudo não tem a pretensão de homogeneizar o ensino de enfermagem gerontogeriátrica mas, com base em pesquisa bibliográfica realizada e nos resultados aqui obtidos, gostariam de sugerir que as escolas de enfermagem, ao abordarem esta temática, considerassem, se ainda não o fazem, alguns ítens que foram sugeridos pela Association for Gerontology in Higher Education conforme descrito a seguir:

- Teorias do envelhecimento

- Alterações normais no envelhecimento

- Problemas mais comuns no envelhecimento

- Habilidades funcionais no idoso

- Políticas públicas relativas à velhice

- Promoção e manutenção da saúde do idoso

- Cuidados prolongados

- Institucionalização

- Atitudes e aspectos éticos relativos a assistência ao idoso

- Variações culturais

- Desenvolvimento profissional

Estes grandes temas foram obtidos por especialistas na área de enfermagem gerontológica a partir de um trabalho desenvolvido no Canadá ${ }^{(9)}$ onde foram citados 49 tópicos considerados fundamentais para que os enfermeiros estivessem habilitados a cuidar de idosos.

Assim, considerando o exposto e outros estudos pesquisados ${ }^{(11,12,19,23,24)}$, relacionam-se a seguir os tópicos de um conteúdo programático, como sugestão para análise das instituições de ensino de enfermagem de nosso país:

- Teorias do envelhecimento

- Desenvolvimento de habilidades na idade madura

- Desenvolvimento da personalidade
- Funçōes cognitivas

- Perdas normais com o envelhecimento

- Envelhecimento saudável e bem-estar na maturidade

- Estratégias de adaptação às mudanças no envelhecimento

- Adaptação psicológica ao envelhecimento

- Aspectos demográficos e epidemiológicos do envelhecimento (futuras tendências)

- Características da população idosa (de cada região) idoso na sociedade

- Respostas governamentais aos idosos

- Aspectos econômicos do envelhecimento

- Força política dos idosos

- Relacionamento familiar

- Aspectos éticos relacionados à assistência ao idoso

- Aspectos étnicos relacionados ao idoso

- Gênero e idosos

- Diversidades em populações idosas

- Atitudes frente ao envelhecimento

- Defesa ao cuidado à saúde dos idosos

- Promoção da saúde

- Recursos comunitários e envelhecimento

- Mudanças físicas normais no envelhecimento

- Problemas de saúde comuns com a avançar dos anos

- Doenças mais freqüentes em idosos

- Farmacologia e idosos

- Nutrição e envelhecimento

- Princípios de reabilitação em geriatria

- Princípios de ativação (catalizadores)

- Cuidados paliativos

- História da enfermagem gerontológica

- Gerontologia: trabalho interdisciplinar

- Pesquisa em gerontologia

- Organização do cuidado à saúde para idosos fragilizados (de risco) processo de enfermagem e o envelhecimento

- Avaliação holística da saúde do idoso

- Avaliação funcional

- Avaliação de saúde mental

- Condutas de avaliação de enfermagem em idosos

- Avaliação do ambiente e seu impacto na independência do idoso

- Suporte social

- Planejamento da terminalidade

- Estratégias para ensinar idosos

- Habilidades requeridas para cuidar de idosos

- Comunicação com idosos com distúrbios cognitivos

- Comunicação com idosos com distúrbios sensoriais

Estes foram os tópicos levantados pelos especialistas em países desenvolvidos. Acreditamos que outros, próprios a nossa cultura e organização social devam ser incluídos tais como: iatrogenia, terapias complementares, assistência domiciliária, instrumentalização de cuidadores de idosos, soluções alternativas à institucionalização. $\mathrm{POSSO}^{(24)}$ publicou recentemente uma experiência neste sentido. 
O prolongamento da vida sempre foi algo que a ciência buscou. Isto vem sendo alcançado gradativamente. No entanto, cabe a todos nós profissionais de saúde dar a estes anos ganhos a dignidade que estes merecem ter. Caso contrário, esta busca pode ter sido em vão.

\section{REFERÊNCIAS BIBLIOGRÁFICAS}

1. ANGERAMI, E.L.S. et al. Estudo comparativo da nomenclatura das matérias e disciplinas do currículo mínimo com a utilizada pelas escolas de enfermagem da região sudeste - Brasil. Rev. Lat. Am. Enf., v. 4, n. 1, p. 31-46, 1996.

2. BERQUO, E.S; LEITE, V.M. Algumas considerações sobre a demografia da população idosa no Brasil. Ci. e Cult. , v. 40 , n. 7 , p. $679-87,1988$.

3. BRASIL. Leis, etc. Lei n. 8.842 de 4 de janeiro de 1994. Dispõe sobre a Política Nacional do Idoso, cria o Conselho Nacional do Idoso e dá outras providências. Diário Oficial da União, Brasília, 5 jan.1994. Seção 1.

4. BRASIL. Ministério da Saúde. Programa de Saúde do idoso: planejamento 1988/92, Brasília, 1988.

5. CAMPEDELLI, M. C. Atuação de enfermagem em geriatria e gerontologia, Rev.Paul.Hosp., v. 31, n. 9 / 10, p. 198-200, 1983.

6. CAVAlCANTI, M.das G.P.de H; SAAD, P.M. Os idosos no contexto da saúde pública In: O IDOSO na grande São Paulo. São Paulo, SEADE, 1990 (Coleção Realidade Paulista).

7. DeLORA, J.R.; MOSES, D.V. Specialty preferences and characteristics of nursing students in baccalaureate programs. Nurs. Res., v. 18, n. 2, p. 137-44, 1969.

8. DUARTE, Y.A.O. A influência da formação acadêmica do enfermeiro na assistência ao idoso. Âmb. Hosp., v. 9, p. 61-6, 1994 .

9. EARTHY, A. A survey of gerontological curriculo in Canada: Generic baccalaureate nursing programs. J. Geront.Nurs., v. 19, n. 12, p. $77-14,1993$.

10. FRIES, J.F.; CRAPO, L.M. Vitality and aging: implications of the retangular curve. San Francisco, W.H.Freeman, 1981.

11. HAIGHT, B.K. et al. Graduate education: creating nursing diagnoses. J. Geront. Nurs., v. 20, n. 2, p. 13-20, 1994.

12. HOLTZEN, V.et al. Baccalaureate curriculum gerontological nursing objectives. J. Geront. Nurs., v. 19, n. 6, p. 35-41, 1993.

13. HOOUVER, S. L. ; SIEGEL, J. A. International demographic trends and perspectives on aging J.Cross-Cult.Geront., n. 1, p. 5-30, 1986.
14.INSTITUTO BRASILEIRO DE GEOGRAFIA E ESTATISTICA Tábuas de mortalidade e sobrevivência brasileiras. Rio de Janeiro, 1981. p. 9-173

15. JACOB FILHO, W; SOUZA, R. R. de. Anatomia e fisiologia do envelhecimento In: CARVALHO FILHO, E. T.de; PAPALÉO NETTO, M. Geriatria: fundamentos, clínica e terapêutica. São Paulo, Atheneu , 1994. cap. 3, p. 31-40.

16. KALACHE, A. et.al. Envelhecimento da população mundial: um desafio novo. Rev.Saúde Públ.; v. 21, n. 3 , p. 200-10, 1987.

17. KINSELLA, K. Dimensiones demográficas y de salud en América Latina y el Caribe In: ORGANIZACION PANAMERICANA DE LA SALUD La atención de los ancianos: um desafio para los años noventa. Washington, 1994. p. 3 - 18.(Publicación científica no. 546).

18. LAURENTI, R. O envelhecimento da população. In: UNIVERSIDADE DE SÃO PAULO. Reitoria. Problemas do idoso: um desafio social. São Paulo, 1984. v.1.

19. MALLIARAKIS, D.R.; HEINE,C. Is gerontological nursing included in baccalaureate nursing programs? J.Geront.Nurs., v. 16, n. 6, p. 4-7, 1990.

20. MARTINEZ, F.M. Educación gerontológica formal. In: ORGANIZACION PANAMERICANA DE LA SALUD. La atención de los ancianos: um desafio para los años noventa. Washington, 1994, p. 404-10. (Publicación científica , n. 546)

21. MENDES, M.M.R. Enfermagem gerontológica e geriátrica: do estado da arte no Brasil a uma proposta para o ensino de graduação. Ribeirão Preto, 1989. Dissertação (Mestrado), Escola de Enfermagem de Ribeirão Preto, Univer-sidade de São Paulo.

22.ORGANIZACION MUNDIAL DE LA SALUD Aplicación de la epidemiologia al estudio de los ancianos: informe de um grupo científico de la OMS Genebra, 1984, (Série Informes Técnicos n. 706).

23.ORGANIZACIÓN PANAMERICANA DE LA SALUD Enfermeria gerontologica: conceptos para la practica. Washington, 1993, p. 59. (Série PALTEX n. 31)

24.POSSO, M.B.S. et al. Enfermagem gerontológica e geriátrica: proposta para o ensino na graduação. Âmb. Hosp., v. 4, p. 25-8, 1996.

25. RAMOS, L.R. et.al. Envelhecimento populacional: uma realidade brasileira. Rev.Saúde Públ. , v. 21, n. 3, p. 211-24, 1987.

26. SCHOUERI JUNIOR., R. Crescimento populacional : aspectos demográficos $\mathrm{e}$ sociais. In: CARVALHO FILHO, E. T. de; PAPALÉO NETTO, M. Geriatria: fundamentos, clínica e terapêutica , São Paulo, Atheneu 1994. cap. 2, p. 9-29.

27. VERAS, R.P. País jovem com cabelos brancos: a saúde do idoso no Brasil, Rio de Janeiro, Relume Dumará, 1994.

28. YURCHUCK, E.R.; BROWER, H.T. Faculty preparation for gerontological nursing. J.Geront.Nurs.; v. 20, n. 1 , p. 17-24, 1994. 